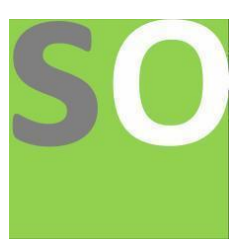

Article title: How the Convolutional Neural Network Work to Identify Numbers?

Authors: Guanlin Jiang[1]

Affiliations: Canada British Columbia International Schools- Chengdu[1]

Orcid ids: 0000-0001-5597-3721[1]

Contact e-mail: jiangguanlin2003@outlook.com

License information: This work has been published open access under Creative Commons Attribution License http://creativecommons.org/licenses/by/4.0/, which permits unrestricted use, distribution, and reproduction in any medium, provided the original work is properly cited. Conditions, terms of use and publishing policy can be found at https://www.scienceopen.com/.

Preprint statement: This article is a preprint and has not been peer-reviewed, under consideration and submitted to ScienceOpen Preprints for open peer review.

DOI: 10.14293/S2199-1006.1.SOR-.PPAIUBJ.v1

Preprint first posted online: 26 May 2021

Keywords: CNN Layers, Computer Vision, Convolutional Neural Networks, Machine Learning, MNIST Handwritten Digit Recognition 


\title{
How the Convolutional Neural Network Work to Identify Numbers?
}

\author{
$1^{\text {st }}$ Guanlin Jiang \\ Canada British Columbia International Schools- Chengdu \\ Chengdu, China \\ jiangguanlin2003@outlook.com
}

\begin{abstract}
In recent years, the Machine Learning field becomes more and more popular. Convolutional Neural Network becomes a famous and beginner level's Neural Network of Machine Learning. This document provides the knowledge of introduces Convolutional Neural Network and Machine Learning, processes and layers of Convolutional Neural Network and using Convolutional Neural Network to training the model to identify numbers.

Index Terms-CNN Layers, Computer Vision, Convolutional Neural Networks, Machine Learning, MNIST Handwritten Digit Recognition
\end{abstract}

\section{INTRODUCTION}

Convolutional Neural Network is a kind of Deep Neural Networks that works in Machine Learning. The most common fields to be used like computer vision, natural language processing, and analysis the visual image information. Convolutional Neural Network is the most popular Deep Learning Neural Network in this world today. The purpose of this paper is to introduce the basic knowledge of Machine Learning and Convolutional Neural Network and how to apply them in real life. This paper will include the introduction of Convolutional Neural Network as a part of Machine Learning, how the Convolutional Neural Network operates and Convolutional Neural Network application on identifying numbers use MNIST Handwritten Digit data set.

\section{Machine Learning and Convolutional Neural NETWORK}

\section{A. What Is Machine Learning}

Machine learning is a part of Artificial Intelligence which is a study of computer algorithms that use data and neural network to let Machine Learning and improve their accuracy [1]. The data set and algorithms are the important elements for Machine Learning. They use algorithms to build the neural network which means build the structures. Then the data set will be label and classify and put into the neural network to train the model which means the algorithms use data to learn, and against their own, practicing the learning and get the max score. Then, researchers can use the model on the raspberry pi, or other electronic devices, or they can add more data sets to training, and improve the accuracy of the models.

The application of Machine Learning includes Computer Vision, Natural Language Processing, and Information Analysis. For example, when a company needs to install a face scanner to help manage employees, they need to collect the employee's face photos to be the data sets. Then they are loading those face data to the computer, and they section off the data. Then programming the algorithms for the neural network and use that to identify patterns which are to train the model. The program of Neural Network can assess and output the results, so people can use the resulting model to run in the electronic devices.

\section{B. What Is Computer Vision}

The Machine Learning is like people to think the things, but Computer Vision is like to envision the things can show in people's brain. When a photo scans or takes a photo by computer, the display will be showing the photo, and perform image classification, those processes are a part of Computer Vision. The image or vision will receive by the computer as the input data. The photo data will be translated to the binary language, which is a language computer can understand, "They first detect pixels, then edges and contours, then whole objects, before eventually producing a final guess about what they're looking at." [2]. After scanning it, the computer will guess what it is. The Computer Vision converts the image into a twodimensional matrix format, which displays to the computer is the binary language. Also, computer vision based on the neural network to accurately identify and classify images, which relies on the models trained by the computer neural network. Like Figure 1 (Cohen, 2020), the image will be divided into many blocks, and each small block will correspond to many two-dimensional matrices. So, this is what computers see.

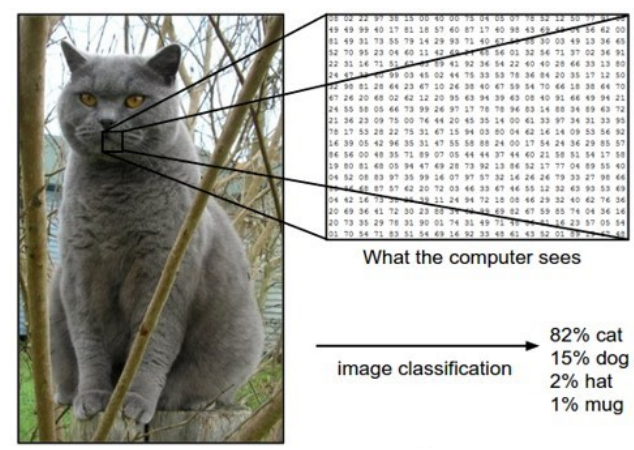

Fig. 1. The example of the computer divided a RGB point to matrices.

Nowadays, Computer Vision application is in facial recognition, and image analysis software, including catching criminals 
and take photos. For example, when people open their computer which can use face to unlock, people put the computer in front of their face, then the camera will be scanning the face and transfer it to binary language, the computer will be analyzing the data by the model and comparing the owner's face data to check and identify is or not owner's face.

\section{What Is Convolutional Neural Network}

"The Convolutional Neural Network is a kind of deep learning neural network which designed for processing structured arrays of data such as images, video, and neural text", which is researched in 1980 by Yann LeCun [3]. The object recognition of the Convolutional Neural Network model imitates the visual processing mechanism of the human brain, each neuron on every layer is connected to the next layer's neurons, which means the whole network uses the layered features. Comparing with "the Back Propagation Model find in 1960, which is a method of fine-tuning the weights of a neural net based on the error rate obtained in the previous epoch" [4]. Convolutional Neural Network comparing with the Back Propagation Model way, which reduce the number of processes for computers to run, and more computing can use in addition the accuracy. After coming out Convolutional Neural Network, the domain of Computer Vision which makes amazing things happen, the training model's accuracy rate is greatly increased, and the error rate is greatly reduced [4]. The application of Convolutional Neural Network uses the training model which is a process on Machine Learning. AI scientists will build a neural network to train their model more accurately and easily to be building and reading the code. The Convolutional Neural Network is the most common for scientists and professional people to use because of the high performance and ease to build.

\section{Processes of Convolutional Neural Network}

Convolutional Neural Network (CNN) include three layers, Convolutional layer, Pooling layer, Fully-connected (FC) layer.

\section{A. Convolutional Layer}

The Convolutional Layer is the first layer of a Convolutional Neural Network. "The convolution layer (CONV) uses filters that perform convolution operations" [5]. This means the Convolution Layer use the filters to get the useful information for whole network. Input data like photos, Convolutional Layer will output some images of size $28 \times 28$ transfer to the next layer, which normally identifies basic features such as straight edges and corners [3]. This layer includes input data, a filter to filter out some noise like the in an animal photo there have a gun in there, also get the important data, and a map of connection the layers. "When an image which made up of a matrix of pixels in 3D, and input to the Convolutional Neural Network, computer understanding the RGB, and the image build by three dimensions, which include a height, width, and depth" [1]. Like Figure 2, the image will be divided into many blocks, and each small block will correspond to many twodimensional matrices. After, the Convolutional Layer will be cut the image to many pieces, and transfer to RGB (which is the pixel of Red, Green, and Blue in computer) of each small image to the binary language, which is display 0 and 1 in the layer, like Figure 1. "While they can vary in size, the filter size is typically a $3 \times 3$ matrix. The filter is then applied to an area of the image" [1]. This means the output matrix is always 3 $\times 3$ matrices, the computer will be calculating every point by the filter and pixels, and those matrices will be transferred to the next layer. The Convolutional Layer is a layer to analyze the input data and convent the image to the number which the computer can understand, help other layers to use the data to training and building a model.

$\begin{array}{lll}1 & 0 & 0 \\ 1 & 0 & 0 \\ 0 & 1 & 1\end{array}$

Fig. 2. Example of Matrix.

\section{B. Pooling Layer}

The Pooling Layer are dividing into two layers, one is Max Pooling, another is Average Pooling. "The functions of Pooling Layer are conducted dimensionality reduction, reducing the number of parameters in the input" [1]. This shows the Pooling layer reducing the size of the image, which means the neural network can reduce some difficulties of calculating. "The pooling layer will then simply perform down-sampling along with the spatial dimensionality of the given input, further reducing the number of parameters within that activation" [6]. This means the Pooling Layer is separates the data from matrix, and decrease the samples of data, just focuses on the features. Also, this layer helps Machine Learning to summarize all the data from Convolutional Layer, and collect the features of the image to processing, which help the model more accurately when using in identify objects [7]. The Pooling layer is the second layer, also like a summarize layer to decrease the difficulties of computing and make the Convolutional Neural Network clearer.

1) Max Pooling: Max Pooling is choosing the most special data like the most feature point in an image from summarizing information and output a map that has the most characteristic parameter from every matrix [7]. Like Figure 3, Matrix 1 after Max Pooling becomes Matrix 2. The Max Pooling is to optimization the data and chooses the high feature parameters to train, and the model output by training use in machines, the process will be to use the test images to identify will focus on the most feature or special part to identify.

2) Average Pooling: The Average Pooling is a process to calculate every matrix average parameter and output a new matrix transfer to the next layer. The point is the Max Pooling just chooses the highest feature one, but the "Average Pooling blends them in" which is using all the parameters which are $2 \times 2$ matrices to get the average value [8]. Like Figure 4, Matrix 3 after Average Pooling, the output will be Matrix 4. The Average Pooling is just using the average value of each 


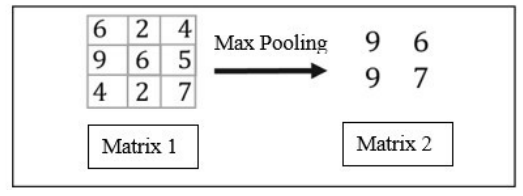

Fig. 3. Example of Max Pooling.

matrix to identify the objects if they can have similar values in the calculating.

\begin{tabular}{|c|c|c|c|c|}
\hline \multirow{3}{*}{$\begin{array}{ll}1 & 4 \\
5 & 9 \\
3 & 6\end{array}$} & \multirow{3}{*}{$\begin{array}{l}2 \\
5 \\
1\end{array}$} & & \multirow{3}{*}{$\begin{array}{l}4.75 \\
5.75\end{array}$} & \multirow{3}{*}{$\begin{array}{c}5 \\
5.25\end{array}$} \\
\hline & & \multirow{3}{*}{ Average Pooling } & & \\
\hline & & & & \\
\hline \multicolumn{2}{|c|}{ Matrix 3} & & \multicolumn{2}{|c|}{ Matrix 4} \\
\hline
\end{tabular}

Fig. 4. Example of Average Pooling.

\section{Fully-connected (FC) Layer}

"Fully-connected Layer is multiplying the input parameters by a matrix with the weight, then adds a bias vector" which is the addition of the neurons' weights in the neural network that do not need the input parameters [9]. In the Fully-connected Layer, input is connected to all neurons. "The Fully-connected Layers will attempt to produce class scores from the activation, to be used for classification. It is also suggested that ReLu may be used between these layers, to improve performance" [6]. This means the functions of neurons are to help the data calculating parameters to connect each point and transfer the calculating result. "The Full-connected Layers which compile the data extracted by last layers to form the final output" [10]. The Fully-connected Layer has three sub-layers- Input Layer, Hidden Layer, and Output Layer. In those layers, there have many units, like the feature units, activation units, and bias units [10]. The Fully-connected Layer will be processing the weight and class recognition by those layers and units. The input layer will get the parameters, and the hidden layers calculate the parameters with weight and bias vector, after the calculation, the result will be transferred to the output layer to be the result. The work of class recognition is the neurons in this layer will catch a certain feature, then the network needs to store the value of each feature, and give the class to the values, also will check it is right or not [11]. After the output layer, the output model file will store all the networks, when people put the model file into other devices, they do not need to be training again, just use is OK. The Fully-connected Layer basically is to classify the value and add the weight to each value to make sure the output model can identify accurately.

\section{Using Convolutional Neural Network to IDENTIFY OBJECTS}

\section{A. Loading Data}

Nowadays, the use of Convolutional Neural Network to get the Machine Learning model is a common thing, because of the clear reading and easy building, data researchers and academic people always use this framework to train the data and build the model. I choose to use Convolutional Neural Network to identify numbers to be the example to explain how. Here, the MNIST Handwritten Digit data set can be used, which is a data set that has many numbers write by other people but offer by Keras which is a Deep Learning framework to be the data set as the input data. We use Python 3 to be the computer programming language, and Jupyter Notebook to be the Python3 Editor based on the web. Importing the Keras deep learning framework, Keras MNIST data set, and the NumPy which is a calculating part for Python. The size of the image needs to be set, $28 \times 28 \times 1$ is the input shape we need. The $\mathrm{x}$-train, $\mathrm{y}$-train, $\mathrm{x}$-test, and y-test need to be setting when we start to load the MNIST data set. "The x-train and $\mathrm{x}$-test are the features you are using as input for the model of train data and test data, y-train and y-test are the expected labels of train data and test data" [12]. In figure 5, $\mathrm{x}$ is the image number data, and $\mathrm{y}$ is the number labels. like the Number 5 image is $\mathrm{x}$, and "Digit: 5" is y label. After loading the data set into the memories, Convolutional Neural Network can be starting to build.

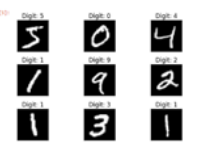

Fig. 5. Example of $x$-train/test and $y$-train/test.

\section{B. Build the Model Training Data}

In this process, Keras be the Convolutional Neural Network builder framework. For this MNIST Convolutional Neural Network, I choose to build two groups of layers in this training, which means the data will be run two times. Building the two-dimensional Convolutional Layers' filters, parameters set are 32 hidden units and 64 hidden units, also using "ReLu". The ReLu equation shows below:

$$
\mathrm{f}(\mathrm{x})=\max (0, \mathrm{x}) \text { or } \mathrm{f}(\mathrm{x})= \begin{cases}0 & x>0 \\ x & x<0\end{cases}
$$

"which is a non-linear activation function can calculate," it to be the activation units and using two times two-dimensional Max Pooling to choose the most feature data to summarize [13]. After, choosing the "Softmax". The Softmax equation shows below:

$$
\operatorname{softmax}\left(\mathrm{z}_{i}\right)=\frac{\exp \left(z_{i}\right)}{\sum_{j} \exp \left(z_{j}\right)}
$$

That is a function that can output a probability distribution, "it can calculates the relative probabilities" [14]. Which means the Softmax function to be the activation unit, calculate the probabilities of the data, and output the results. In this process, we need to build the Model of the Convolutional Neural Network and decide the layers want to set. After the Convolutional Neural Network model built, the input data can be training. Setting the epoch which is how many times people want the Network training and setting the accuracy and loss for training and test. When the program running, after training, 
the model file will be output. "The trained model needs to be evaluated in terms of performance" [12]. If the performance reaches $99 \%+$, which means the model is trained well for prediction. So, this file people can put loading in the programs, and use it in any electric devices.

\section{Conclusion}

Convolutional Neural Network is a part of Machine Learning as an Artificial Intelligence algorithms framework and it applies to Computer Visions and Neural Language Processing, which can put a lot of data make machine training (learning) to get a more accurate result. This Network includes 3 layers- Convolutional Layer, Pooling Layer, and Fullyconnected Layer which is also following the processes. Using Convolutional Neural Network to identify numbers to be an example of how to build algorithms and train a Machine Learning model. The application of identification number using Convolutional Neural Network to training the MNIST Handwritten Digit data set, which shows how Convolutional Neural Network use and work.

\section{REFERENCES}

[1] By: IBM Cloud Education, "What is Machine Learning?," IBM. [Online]. Available: https://www.ibm.com/cloud/learn/machine-learning. [Accessed: 25-Mar-2021]

[2] K. Hartnett and substantive Quanta Magazine moderates comments tonbsp;facilitate an informed, "Computers and Humans 'See' Differently. Does It Matter?," Quanta Magazine. [Online]. Available: https://www.quantamagazine.org/computers-and-humans-seedifferently-does-it-matter-20190917. [Accessed: 25-Mar-2021]

[3] T. Wood, "Convolutional Neural Network," DeepAI, 17-May-2019 [Online]. Available: https://deepai.org/machine-learning-glossary-andterms/convolutional-neural-network. [Accessed: 25-Mar-2021]

[4] K. D. Foote, "A Brief History of Deep Learning," DATAVERSITY, 31 Jan-2017. [Online]. Available: https://www.dataversity.net/brief-historydeep-learning/. [Accessed: 25-Mar-2021]

[5] A. Amidi and S. Amidi, "Convolutional Neural Networks cheatsheet Star," CS 230 - Convolutional Neural Networks Cheatsheet. [Online]. Available: https://stanford.edu/ shervine/teaching/cs-230/cheatsheetconvolutional-neural-networks. [Accessed: 25-Mar-2021]

[6] K. O'Shea, R. Nash, "An Introduction to Convolutional Neural Networks," arXiv:1511.08458 [cs], Dec. 2015

[7] S. Hosla, "CNN: Introduction to Pooling Layer," GeeksforGeeks, 26-Aug-2019. [Online]. Available: https://www.geeksforgeeks.org/cnnintroduction-to-pooling-layer/. [Accessed: 25-Mar-2021]

[8] Christian, "What are Max Pooling, Average Pooling, Global Max Pooling and Global Average Pooling?," MachineCurve, 30-Jan-2020. [Online]. Available: https://www.machinecurve.com/index.php/2020/01/30/what-are-maxpooling-average-pooling-global-max-pooling-and-global-averagepooling/. [Accessed: 25-Mar-2021]

[9] Mathwork, "fullyConnectedLayer," Fully connected layer - MATLAB, 2021. [Online]. Available: https://www.mathworks.com/help/deeplearning/ref/nnet.cnn.layer.fullyconnectedlayer.html. [Accessed: 25-Mar-2021]

[10] S. P. Singh, "Fully Connected Layer: The brute force layer of a Machine Learning model,' OpenGenus IQ: Learn Computer Science, 02Mar-2019. [Online]. Available: https://iq.opengenus.org/fully-connectedlayer/. [Accessed: 25-Mar-2021]

[11] SuperDataScience Team, SuperDataScience, 18-Aug-2018. [Online]. Available: https://www.superdatascience.com/blogs/convolutionalneural-networks-cnn-step-4-full-connection. [Accessed: 25-Mar-2021]

[12] S. Mahapatra, "A simple 2D CNN for MNIST digit recognition," 22-May-2018. [Online]. Available: https://towardsdatascience.com/asimple-2d-cnn-for-mnist-digit-recognition-a998dbc1e79a. [Accessed: 09-Apr-2021]
[13] DeepAI, "ReLu," DeepAI, 17-May-2019. [Online]. Available: https://deepai.org/machine-learning-glossary-and-terms/relu. [Accessed: 07-May-2021]

[14] Shipra SaxenaShipra is a Data Science enthusiast, "Softmax: What is Softmax Activation Function: Introduction to Softmax," Analytics Vidhya, 06-Apr-2021. [Online]. Available: https://www.analyticsvidhya.com/blog/2021/04/introduction-to-softmaxfor-neural-network/. [Accessed: 07-May-2021] 